\title{
Overview on Auto inflammatory Bone Disorders: From Pathogenesis to Clinical Manifestations
}

\author{
Marino $\mathbf{A}^{1,2 *}$ \\ 1PhD student, University of Florence, Florence, Italy \\ ${ }^{2}$ Department of Pediatrics, Desio Hospital, ASST Monza, Italy \\ Italy

\section{Chronic Recurrent Multifocal Osteomyelitis (CRMO)}

*Corresponding author: Achille Marino, PhD student, University of Florence, Florence,
Chronic Recurrent Multifocal Osteomyelitis (CRMO) is a rare disease occurring in childhood. It is characterized by insidious onset and variable clinical manifestations that may mimic infections or malignancies [1]. Since CRMO may be neither multifocal nor recurrent, some authors suggest replacing it with the term chronic nonbacterial osteomyelitis (CNO) that includes different disorders with sterile bone inflammation [2]. Synovitis, acne, pustulosis, hyperostosis and osteitis syndrome (SAPHO) is the adult form of CRMO with prominent skin manifestations [3]. CRMO is frequently associated with other inflammatory diseases such as psoriasis and inflammatory bowel disease [4]. CRMO pathogenesis is largely unknown; however, an unbalance towards proinflammatory cytokines has been demonstrated $[5,6]$. Recently a South Asian child with CRMO and psoriasis has been found harboring an autosomal recessive mutation of FBLIM1 gene [7].

Clinical manifestations may include systemic features such as low-grade fever, pain, slight malaise and fatigue [8]. Bone lesions are often multifocal, mostly in long bones; whereas spinal involvement may lead to severe complications. Laboratory tests are unspecific showing a mild elevation inflammatory markers. Conventional radiography can show the presence of osteolytic lesions typical of advanced disease, whereas early bone lesions may be detected by whole-body MRI (STIR sequences) [9]. Since CRMO is a diagnosis of exclusion, bone biopsies are useful to exclude malignancies showing unspecific inflammatory infiltrate. Recently, very high IL-6 serum levels ( $\geq 17 \mathrm{ng} / \mathrm{ml}$ ) have been detected in CRMO patients along with low levels of the eosinophil attracting chemokine (CCL11/eotaxin) (<110 ng/ml). The combination of both biomarkers has been proposed to discriminate patients with inflammatory diseases from healthy controls [10].

The treatment of CRMO is based on nonsteroidal antiinflammatory drugs (NSAIDs) that represent first-line therapy [1]. NSAIDs lead to pain control and prevention of bone lesions in a large portion of patients [1]. Bisphosphonates are used especially for spinal lesions in the absence of systemic features. Moreover, a trial on pamidronate in CRMO patients is now ongoing (NCT02594878). TNF- $\alpha$ inhibitors are commonly used when first-line therapies fail. The role of TNF- $\alpha$ in osteoclastogenesis and bone resorption is well known, thus anti-TNF $\alpha$ agents are very useful in these patients [11].

Childhood Arthritis and Rheumatology Research Alliance (CARRA) have recently issued a consensus treatment plan for CRMO. After NSAIDs failure (defined as the persistence of symptoms following at least 4 weeks of continuous treatment) one of the following treatments is recommended: methotrexate or sulfasalazine, anti-TNF $\alpha$ agents with or without methotrexate, and bisphosphonates [12].

\section{Deficiency of IL-1 Receptor Antagonist (DIRA)}

Patients with deficiency of IL-1 receptor antagonist (DIRA) show both skin and bone involvement along with systemic inflammation. Recessive mutation of IL1RN gene causes loss of IL-1 $\beta$ and IL-1 $\alpha$ physiological inhibition 


\section{Journal of Orthopedics \& Bone Disorders}

[13]. Pustular lesions appear within the first months of life and are associated with inflammatory markers rising; later on DIRA patients develop osteitis and periostitis [13]. Bone lesions show neutrophilic infiltrate at biopsies. Widening of the anterior rib ends and periostitis of long bones are classically described in these patients $[13,14]$. The exogenous IL- 1 receptor antagonist, anakinra, is able to neutralize both IL- $1 \alpha$ and IL- $1 \beta$ and is successfully employed in DIRA patients [13.]

\section{Majeed Syndrome}

Majeed syndrome, characterized by sterile osteomyelitis, dyserythropoietic anemia and neutrophilic dermatosis. It is a monogenic bone autoinflammatory disease due to loss of function mutation of LPIN2 inherited in an autosomal recessive pattern. The protein LIPIN2 is involved in lipid metabolism [15]. Disease onset is within 2 years of age and is usually associated with fever attacks and growth disturbances along with joint contractures. Some Majeed patients do not show skin manifestations. The efficacy of IL-1 blocking agents has indirectly confirmed the pathogenesis of Majeed syndrome [16].

\section{Cherubism}

Cherubism is due to heterozygous mutations of SH3 binding protein 2 (SH3BP2) gene with variable penetrance and phenotypic manifestation within the same family. Furthermore, de novo mutations are also described [17]. SH3BP2 plays a key role in osteoclast activation fostering bone resorption [18]. These patients show osteolytic lesions of mandibles very early in life; the following fibrotic tissue deposition and bone remodeling give to patients with cherubism the typical aspect with puffy cheeks [19]. Ribs may be the other location of disease [19]. Cherubism treatment is based on case reports in which adalimumab and bisphosphonates have been both used [20, 21].

\section{References}

1. Girschick H, Finetti M, Orlando F, Schalm S, Insalaco A, et al. (2018) The multifaceted presentation of chronic recurrent multifocal osteomyelitis: a series of 486 cases from the Eurofever international registry. Rheumatology (Oxford) 57(8): 1504.

2. Ben-Chetrit E, Gattorno M, Gul A, Kastner DL, Lachmann HJ, et al. (2018) Consensus proposal for taxonomy and definition of the autoinflammatory diseases (AIDs): a Delphi study. Ann Rheum Dis 77(11): 1558-1565.

3. Hayem G, Bouchaud-Chabot A, Benali K, Roux S, Palazzo E, et al. (1999) SAPHO syndrome: a long-term follow-up study of 120 cases. Semin Arthritis Rheum 29(3): 159-171.

4. Wipff J, Costantino F, Lemelle I, Pajot C, Duquesne A, et al. (2015) A large national cohort of French patients with chronic recurrent multifocal osteitis. Arthritis Rheumatol 67(4): 1128-1137.

5. Scianaro R, Insalaco A, Bracci Laudiero L, De Vito R, Pezzullo M, et al. (2014) Deregulation of the IL-1b axis in chronic recurrent multifocal osteomyelitis. Pediatr Rheumatol Online J 17(12): 30

6. Hofmann SR, Kubasch AS, Ioannidis C, Rosen-Wolff A, Gir- schick HJ, et al. (2015) Altered expression of IL10 family cytokines in monocytes from CRMO patients result in enhanced IL-1b expression and release. Clin Immunol 161: 300-307.

7. Cox AJDBW, Laxer RM, Velez G, Bing X, Finer AL, et al. (2017) Recessive coding and regulatory mutations in FBLIM1 underlie the pathogenesis of Chronic Recurrent Multifocal Osteomyelitis (CRMO). PLoS One 12(3): e0169687.

8. Alshammari A, Usmani S, Elgazzar AH, Ashkanani RA (2013) Chronic Recurrent Multifocal Osteomyelitis in Children: A Multidisciplinary Approach is needed to establish a Diagnosis. World J Nucl Med 12(3): 120123.

9. Fritz J, Tzaribatchev N, Claussen C, Carrino JA, Horger MS (2009) Chronic recurrent multifocal osteomyelitis: comparison of whole-Body MR imaging with radiography and correlation with clinical and laboratory data. Radiology 252(3): 842-851.

10. Hofmann SR, Böttger F, Range U, Lück C, Morbach H, et al. (2017) Serum Interleukin-6 and CCL11/Eotaxin May Be Suitable Biomarkers for the Diagnosis of Chronic Nonbacterial Osteomyelitis. Front Pediatr 5: 256

11. Eisenstein EM, Syverson GD, Vora SS, Williams CB (2011) Combination therapy with methotrexate and etanercept for refractory chronic recurrent multifocal osteomyelitis. J Rheumatol 38(4): 782-783. 


\section{Journal of Orthopedics \& Bone Disorders}

12. Zhao Y, Wu EY, Oliver MS, Cooper AM, Basiaga ML, et al. (2018) Consensus Treatment Plans for Chronic Nonbacterial Osteomyelitis Refractory to Nonsteroidal Antiinflammatory Drugs and/or With Active Spinal Lesions. Arthritis Care Res (Hoboken) 70(8): 1228-1237.

13. Aksentijevich I, Masters SL, Ferguson PJ, Dancey P, Frenkel J, et al. (2009) An autoinflammatory disease with deficiency of the interleukin-1-receptor antagonist. N Engl J Med 360(23): 2426-2437.

14. Jesus AA, Osman M, Silva CA, Kim PW, Pham TH, et al. (2011) A novel mutation of IL1RN in the deficiency of interleukin-1 receptor antagonist syndrome: description of two unrelated cases from Brazil. Arthritis Rheum 63(12): 4007-4017.

15. Donkor J, Sariahmetoglu M, Dewald J, Brindley DN, Reue K (2007) Three mammalian lipins act as phosphatidate phosphatases with distinct tissue expression patterns. J Biol Chem 282(6): 3450-3457.

16. Herlin T, Fiirgaard B, Bjerre M, Kerndrup G, Hasle H, et al. (2013) Efficacy of anti-IL-1 treatment in Majeed syndrome. Ann Rheum Dis 72(3): 410-413.
17. Ueki Y, Tiziani V, Santanna C, Fukai N, Maulik C, et al. (2001) Mutations in the gene encoding c-Abl-binding protein SH3BP2 cause cherubism. Nat Genet 28(2):125-126.

18. GuezGuez A, Prod'homme V, Mouska X, Baudot A, Blin-Wakkach C, et al. (2010) 3BP2 Adapter protein is required for receptor activator of NFkappaB ligand (RANKL)-induced osteoclast differentiation of RAW264.7 cells. J Biol Chem 285(27): 20952-20963.

19. Papadaki ME, Lietman SA, Levine MA, Olsen BR, Kaban LB, et al. (2012) Cherubism: best clinical practice. Orphanet J Rare Dis 7(S1): S6.

20. Hero M, Suomalainen A, Hagström J, Stoor P, Kontio R, et al. (2013) Anti-tumor necrosis factor treatment in cherubism-clinical, radiological and histological findings in two children. Bone 52(1): 347-353.

21. Pagnini I, Simonini G, Mortilla M, Giani T, Pascoli L, et al. (2011) Ineffectiveness of tumor necrosis factoralpha inhibition in association with bisphosphonates for the treatment of cherubism. Clin Exp Rheumatol 29(1): 147. 